\title{
Evaluación de métodos químicos y mecánicos para promover la germinación de semillas y producción de fosforitos en café (Coffea arabica) var. Catuaí Rojo
}

\author{
Evaluation of chemical and mechanical methods to promote the seed germination \\ and production of match seedlings of coffee (Coffea arabica) var. Catuai Rojo
}

\author{
Martín Coa Urbaez ${ }^{1}$, Jesús Rafael Mendez Natera ${ }^{2 *}$, \\ Ramón Silva Acuña ${ }^{3}$ y Sol Mundarain Padilla ${ }^{1}$
}

\begin{abstract}
RESUMEN
El ensayo se condujo a cielo abierto, en las instalaciones de la Escuela Técnica Robinsoniana Aragua de Maturín, ubicada en la capital del municipio Piar del estado Monagas, con el propósito de evaluar el efecto de diferentes tratamientos de escarificación de semillas de café sobre la germinación y formación de los fosforitos. Se emplearon semillas seleccionadas de café, de la variedad Catuaí Rojo, adquiridas en el Instituto Nacional de Investigaciones Agrícolas. Se utilizó como sustrato arena lavada de río, desinfectada con agua hirviendo, contenida en bandejas tipo jardineras de 12 x 40 x $15 \mathrm{~cm}$. Los tres tratamientos con la escarificación mecánica fueron sin inmersión en agua e inmersión durante 24 y 48 horas; los seis tratamientos con escarificación química empleando ácido de batería (Copper $\left.{ }^{\circledR}\right)$ y ácido muriático (Opinß) durante 10, 20 y 30 minutos de inmersión; además de dos tratamientos con inmersión en agua durante 24 y 48 horas y el tratamiento testigo como lo realiza el productor cafetalero (sin escarificación e inmersión). Los 12 tratamientos evaluados en el ensayo constaron de cuatro repeticiones y se organizaron en el diseño experimental de bloques al azar. La unidad experimental estuvo constituida por 50 semillas. Las evaluaciones se realizaron a los $25,30,40$ y 50 días después de soterrada la semilla en las jardineras. Los tratamientos donde las semillas de café se sometieron a escarificación mecánica más inmersión en agua durante 24 y 48 horas presentaron los mayores valores de germinación y formación de fosforitos, en los cuales se constató que más del $60 \%$ de la germinación ocurrió a los 25 días y la formación de fosforitos ocurrió a los 30 días de soterradas las semillas. Los tratamientos de escarificación química empleando los productos comerciales con ácido muriático y ácido de batería no ofrecieron ventajas con relación al testigo.
\end{abstract}

Palabras clave: Técnicas de escarificación, ácido sulfúrico, ácido clorhídrico, inmersión en agua.

\begin{abstract}
The experiment was carried out in the open at the Escuela Técnica Robinsoniana Aragua de Maturin, located in the capital of Piar municipality Monagas State in order to evaluate the effect of different coffee seed scarification treatments on germination and formation of match seedlings. Selected coffee seeds of variety Catuai Red from Instituto Nacional de Investigaciones Agrícolas were used. Washed river sand was used as a substrate, it was disinfected with boiling water and put in garden type trays $12 \times 40$ $x 15 \mathrm{~cm}$. The three treatments with mechanical scarification were without water immersion and immersion for 24 and 48 hours, all six treatments employing chemical stratification with battery acid (Copper $®$ ) and muriatic acid (Opin $®)$ for 10, 20 and 30 minutes of immersion; moreover, two treatments with immersion in water for 24 and 48 hours and the control treatment was the used one for coffee producer (without scarification and immersion). The experimental design was a randomized complete block with 12 treatments and four replications. The experimental unit consisted of 50 seeds. Evaluations were carried out at 25, 30, 40 and 50 days after sowing (DAS). Treatments where coffee seeds were subjected to mechanical scarification and water immersion for 24 and 48 hours showed the highest values of germination and formation of match seedlings, in which, it was found that over $60 \%$ of germination occurred at 25 days and formation of match seedlings occurred at 30 DAS. Chemical scarification treatments using commercial products with muriatic acid and battery acid did not have any advantage in relation to the control.
\end{abstract}

Key words: Scarification techniques, sulphuric acid, chlorhidric acid, water inmersion.

1 Postgrado en Agricultura Tropical, Núcleo Monagas, Universidad de Oriente. Campus Juanico, Maturín, 6201, estado Monagas. Venezuela.

2 Departamento de Agronomía, Escuela de Ingeniería Agronómica, Universidad de Oriente. Avenida Universidad Campus Los Guaritos, Maturín.

3 Instituto Nacional de Investigaciones Agrícolas, Maturín.

* Autor por correspondencia: jmendezn@ cantv.net

Fecha de Recepción: 8 Julio, 2013.

Fecha de Aceptación: 21 Noviembre, 2013. 


\section{Introducción}

Para Venezuela el cafeto reviste gran importancia, debido a que es cultivado principalmente por pequeños agricultores con amplia tradición en este rubro, y del cual dependen casi exclusivamente para su sustento; asimismo, las zonas cafetaleras desempeñan un rol importante en la conservación del ambiente y los recursos naturales, al proteger los suelos en pendientes y contribuir con la sustentabilidad de la biodiversidad y de los cuerpos de aguas.

La semilla de café es de forma semicircular, está formada por la almendra o la semilla sin pergamino, dura, de color verdoso constituida por un albumen córneo cuyo tejido contiene almidón, sustancias grasas, azúcares, sacarosa, taninos, cafeína, etc. En un extremo de la semilla se encuentra un embrión de raicilla cónica y cotiledones cordiformes. Las dimensiones están comprendidas entre 8,5 y 12,17 mm (MEA, 1983; Rincón, 1982). La semilla madura, sana, bien constituida puede germinar desde su cosecha si se coloca en un medio que presente condiciones satisfactorias: humedad, calor y aire (Urbaneja y Quijada, 2006); sin embargo, muchas semillas viables son incapaces de germinar inmediatamente después de madura, aunque se les coloque en condiciones favorables para la germinación. Esta característica es denominada latencia o germinación diferida, y una de sus causas es la impermeabilidad del tegumento (Sanabria et al., 2004).

Aparentemente, la latencia es un mecanismo de supervivencia ante la presencia de determinadas condiciones climáticas: temperaturas muy bajas, alternancias de épocas secas y húmedas y climas desérticos (Sanabria et al., 2004). Solomon et al. (2001) expresan que se desconocen las causas exactas del fenómeno de latencia, por otro lado, cuando la latencia se debe a condiciones de la testa, el letargo termina en el momento en que esta se agrieta o debilita por acciones mecánicas o químicas o por efecto del ambiente o acción química (Madueño et al., 2006); sin embargo, las semillas de café carecen de periodo de latencia, aunque la presencia del endocarpio atrasa la germinación. Semillas con endocarpio presente germinan entre los 50 y 75 días, siendo que su remoción acelera la germinación en 20 días (Valio, 1980).

Moreno Martínez (1996) señala que la germinación se define como la emergencia y desarrollo de aquellas estructuras esenciales que provienen del embrión y que manifiestan la capacidad de la semilla para producir una planta normal bajo condiciones favorables. La aparición de la radícula o raíz embrionaria es el evento que evidencia el fenómeno de la germinación, el siguiente en emerger es el hipocotilo, el cual lo hace en forma de gancho invertido acción empleada para proteger la delicada punta del tallo (Solomon et al., 2001). Para que se desarrollen estas fases se requiere de una serie de procesos que comienzan con la imbibición y culmina con la emergencia de la plántula a través de las cubiertas. La germinación es el desarrollo del germen contenido en las semillas. Varios factores como temperatura, agua, oxígeno y presencia de la luz influyen para que una semilla germine o no (Izco et al., 1997).

Las semillas maduras (secas) de café tienen una germinación lenta y asincrónica, lo que hace que sea difícil obtener plántulas que son ideales para el establecimiento de cultivos de café y la producción de café y se ha realizado poco trabajo para entender la germinación de las semillas de café y su regulación. Tales estudios son esenciales para el mejoramiento de las prácticas agrícolas y el desarrollo adicional de la producción de café. (Da Silva, 2002, Eira et al., 2006).

Una vez que son soterradas las semillas de café absorben agua del sustrato y la semilla comienza a crecer. La absorción de agua por la semilla desencadena una secuencia de cambios metabólicos, que incluyen la respiración, la síntesis proteica y la movilización de reservas. A su vez la división y el alargamiento celular en el embrión provoca la rotura de las cubiertas seminales, que generalmente se produce por la emergencia de la radícula que sale fuera del pergamino, esto se registra de cuatro a seis semanas, después emerge el hipocotilo en forma de "bastoncito" y su alargamiento arrastra fuera de tierra a la semilla, siempre envuelta en un endocarpio, al mismo tiempo, las hojas cotiledonales. Estas no salen de la cubierta de pergamino, sino que permanecen en su interior alimentándose de las sustancias de reserva de la almendra de la semilla. Poco tiempo después aproximadamente entre 10 y 15 días se evidencia la verticalidad del hipocotilo (fosforito) más tarde esta ligera cáscara se desprende y dos hojas cotiledónicas se expanden (chapolas) en un periodo de 19 a 30 días para un tiempo estimado del proceso entre 50 y 75 días (MEA, 1983; Rincón, 1982). 
La escarificación, proceso de raspar o mellar el epispermo (físicamente con una herramienta o químicamente con un ácido) antes de la siembra induce a estas semillas a germinar. En la naturaleza, la escarificación ocurre cuando estas semillas pasan por el aparato digestivo de algunos animales o cuando parte del epispermo es digerido por bacterias (Solomon et al., 2001). Existen esencialmente dos tipos: la escarificación mecánica y la química. La escarificación mecánica puede hacerse por medio de un escarificador eléctrico o con cualquier elemento abrasivo que corte, perfore o raspe el tegumento; sin embargo, no es práctico de utilizar para grandes cantidades de semillas (Sanabria et al., 2004).

El método de escarificación química se realiza por inmersión de las semillas en ácido sulfúrico concentrado por un tiempo, el cual depende de cada especie; otro ácido, el clorhídrico industrial, conocido en forma bruta como ácido muriático, podría constituirse potencialmente en una herramienta válida para el proceso de escarificación química de las semillas de café.

El hecho de que las semillas de café tengan un periodo de germinación muy prolongado ha provocado que muchos caficultores justifiquen la compra de plántulas a los viveristas de la zona, por supuesto esto genera aumento de los costos de producción en el primer año de fundación o renovación de las plantaciones del rubro, lo cual es negativo, toda vez que la mano de obra para este cultivo es muy elevada por ser realizadas manualmente; además, las condiciones agroclimáticas en donde se cultiva requieren de mucha fuerza de trabajo manual.

De lo anterior se desprende la dificultad económica que ocasiona el prolongado periodo de germinación que demandan las semillas de cafeto, la cual es provocada entre otras cosas, por la presencia de un endocarpio que retarda su imbibición y prolonga la germinación. En función de lo antes expuesto, el objetivo de la presente investigación fue evaluar el proceso de la escarificación química y mecánica, sobre la germinación y formación de fosforitos para la producción de viveros.

\section{Materiales y métodos}

\section{Ubicación del ensayo, material biológico y sustrato}

El ensayo se realizó en las instalaciones de la Escuela Técnica Robinsoniana Aragua de Maturín, ubicada en la capital del municipio Piar del estado Monagas. Se utilizaron semillas de café (Coffea arabica) var. "Catuaí Rojo", procedentes del Instituto Nacional de Investigaciones Agrícolas, Estación Experimental Local Caripe (INIA-Caripe), destinada para la comercialización como semillas seleccionadas. Como sustrato se empleó arena lavada de río que previamente fue sometida a desinfección con agua caliente a $100^{\circ} \mathrm{C}$. Las semillas se soterraron el día siguiente del tratamiento térmico del sustrato y se colocaron a $1 \mathrm{~cm}$ de profundidad. El riego se efectuó de forma interdiaria.

\section{Tratamientos, diseño experimental y análisis estadístico}

En el ensayo se evaluaron 12 tratamientos, se utilizó el diseño experimental de bloques al azar con cuatro repeticiones. La unidad experimental estuvo constituida por 50 semillas, soterradas en jardineras plásticas de 12 × 40 x $15 \mathrm{~cm}$, para un total de 48 , las cuales fueron mantenidas a cielo abierto.

Los 12 tratamientos consistieron de la evaluación de tres técnicas de escarificación y se colocó un testigo donde la semilla se soterra sin retirar el pergamino o endocarpio y sin haber sido sumergida en agua. Las variables fueron examinadas empleando análisis de varianza y la comparación entre los tratamientos se realizó mediante la prueba de la Mínima Diferencia Significativa (MDS) al 5\% de significación. En el caso de los porcentajes las variables se transformaron mediante la fórmula:

$$
\mathrm{p}^{\prime}=\sqrt{\frac{X+\frac{3}{8}}{n+\frac{3}{4}}}
$$

Donde:

$\mathrm{X}=$ Porcentaje

$\mathrm{n}$ = Tamaño de la muestra la cual compone cada porcentaje

\section{Técnica 1. Escarificación mecánica con y sin inmersión en agua}

Las semillas se escarificaron de forma física empleando un rallador metálico utilizado comúnmente para el rallado casero (queso, zanahoria, pan, etc.) y se emplearon guantes en las manos para 
evitar heridas durante la actividad de fricción de las semillas, a fin de promover la remoción del pergamino. A continuación una parte de las semillas se sometieron a inmersión en agua durante 24 horas, y la otra parte a 48 horas. Se colocó un testigo que no recibió inmersión en agua. Posteriormente, una vez cumplidos los tiempos de inmersión fueron soterradas en las jardineras.

\section{Técnica 2. Inmersión en agua durante 24 y 48 horas}

Estos tratamientos consistieron en sumergir las semillas en agua sin removerles el pergamino o endocarpio por un tiempo de 24 y 48 horas, seguidamente se soterraron para su evaluación.

\section{Técnica 3. Escarificación química con dos productos comerciales}

En este ensayo las semillas se escarificaron por inmersión en ácido de baterías (ácido sulfúrico al 20\%) y ácido muriático (ácido clorhídrico al $10 \%$ ) durante 10, 20 y 30 minutos. Los productos comerciales empleados fueron: ácido de batería Copper® y el limpiador ácido muriático Opin ${ }^{\circledR}$, estos se seleccionaron debido a su facilidad de adquisición y su bajo costo en el mercado comparado con aquellos productos de laboratorio. Concluidos los periodos de inmersión en cada uno de los ácidos, las semillas se lavaron en agua corriente, potable, por cinco minutos, en condiciones de laboratorio, con el objetivo de remover los residuos de ellos y posteriormente se secaron a la sombra y fueron soterradas.

\section{Técnica 4. Testigo con pergamino y sin inmersión en agua (usada por el productor)}

La semilla se soterró en el sustrato sin retirar el pergamino o endocarpio y sin inmersión en agua (Amaya, et al., 1988; Medina et al., 1987) o ácido y sin escarificación mecánica

\section{Definiciones operativas}

Se consideró como semilla germinada aquella donde el hipocotilo emergía en forma de "bastoncito" a la superficie (Solomon et al., 2001).

Fosforito: etapa en la fenología del cafeto durante la fase de vivero. Es la estructura que disminuye los efectos del estrés y garantiza la supervivencia de la plántula y es ideal para el transplante en las bolsas de polietileno.

Chapola: etapa en la fenología del cafeto en condiciones de viveros. La plántula presenta las hojas cotiledonales extendidas.

Las semillas germinadas se cuantificaban diariamente durante un periodo de 50 días. $\mathrm{La}$ formación del "fosforito" se evaluó diariamente durante 24 días consecutivos después del inicio de su formación hasta el día 50 .

\section{Variables evaluadas}

Porcentaje de germinación y porcentaje de formación de "fosforitos": Las evaluaciones se realizaron a los 25, 30, 40 y 50 días después de soterrada la semilla en las jardineras

Índice de la velocidad de germinación e índice de la velocidad de formación de fosforitos: se calculó mediante la siguiente fórmula:

$$
\begin{aligned}
\mathrm{IVG}= & \left.\mathrm{N}_{1} * 4+\mathrm{N}_{2} * 4+\ldots+\mathrm{Ni}^{*} 4\right) / \mathrm{Ti} \\
& (\text { Khan y Ungar, 1984) }
\end{aligned}
$$

Índice de germinación e índice de formación de "fosforitos: se calculó mediante la siguiente fórmula:

$$
\mathrm{IG}=\underset{\text { (Maguire, 1962) }}{\left(\mathrm{N}_{1} / \mathrm{T}_{1}+\mathrm{N}_{2 /} \mathrm{T}_{2}+\ldots+\mathrm{Ni} / \mathrm{Ti}\right)}
$$

Número medio de días a total germinación y total formación de "fosforitos": se calculó mediante la siguiente fórmula:

$$
\begin{gathered}
\mathrm{NMD}=\left(\mathrm{N}_{1} * \mathrm{~T}_{1}+\mathrm{N}_{2} * \mathrm{~T}_{2} . .+\mathrm{NiTi}\right) / \mathrm{n} \\
(\text { Hartman } \text { et al. }, 1993)
\end{gathered}
$$

Donde:

$\mathrm{N}=$ Número de semillas germinadas dentro de los intervalos de tiempo consecutivos,

$\mathrm{T}=$ Tiempo transcurrido entre el inicio de la prueba y el final del intervalo $\mathrm{n}=$ Número total de semillas germinadas.

\section{Resultados y discusión}

Todas las variables estudiadas porcentaje de germinación (POG) y formación de fosforitos (PFOF), días a total germinación (DTG), índice de velocidad de germinación (IVG), índice de germinación (IG), 
días a total formación de fosforitos (DTFF), índice de la velocidad de formación de fosforitos (IVFF) e índice de la formación de fosfóricos (IFF) tuvieron diferencias estadísticas significativas entre los tratamientos (Tablas 1 y 2).

\section{Porcentaje de germinación}

Los tratamientos de escarificación mecánica con inmersión en agua durante 24 y 48 horas presentaron el mayor porcentaje de germinación en todos los tiempos de evaluación (25, 30, 40 y 50 días después de soterradas las semillas) (Tabla 3), seguido por la escarificación mecánica sin inmersión en agua, el cual presentó porcentajes de germinación superiores al resto de los tratamientos. Los tratamientos de inmersión en agua, ácido de batería y ácido muriático presentaron porcentajes de germinación similares al testigo en todos los tiempos de evaluación (Tabla 3).

Los resultados obtenidos están en concordancia con los señalados por Guevara y Ramiro (1992) sobre la ventaja de la germinación cuando es eliminado el pergamino y la dificultad que él representa al limitar la difusión del oxígeno hacia el embrión, lo que impide su crecimiento. Para las evaluaciones del porcentaje de germinación durante los 30 y 40 días de soterradas las semillas la comparación de medias ratifica que los tratamientos correspondientes a ESC-MEC-IMN/24h y ESC-MEC-INM/48h fueron superiores al resto de los tratamientos. $\mathrm{La}$ evaluación realizada el día 49 después de la siembra en todos los tratamientos se evidenciaron mejoras en los valores relativos de germinación, pero cuando estos se confrontan con los tratamientos ESC-MECIMN/24h y ESC-MEC-INM/48h sus valores medios y porcentuales son muy superiores al resto, lo cual sugiere la importancia que tiene la remoción del endocarpio de las semillas y la inmersión en agua; sin embargo, el día 49 los tratamientos ESC-MEC e inmersión en ácido de batería durante 30 minutos (INM-Ac.BAT/30s) experimentan un aumento en sus valores, pero no difieren estadísticamente. En

Tabla 1. Análisis de varianza para el porcentaje de germinación (POG) y porcentaje de formación de fosforitos (PFOF) en el cultivo de café (Coffea arabica) var. Catuaí Rojo en Aragua de Maturín, estado Monagas, Venezuela. Datos transformados mediante $\mathrm{p}^{\prime}=\sqrt{(\mathrm{x}+3 / 8) /(\mathrm{n}+3 / 4)}$.

\begin{tabular}{lcccccccc}
\hline \multirow{2}{*}{$\begin{array}{l}\text { Fuente de } \\
\text { Variación }\end{array}$} & GL & \multicolumn{9}{c}{ Cuadrados medios } \\
\cline { 3 - 9 } & & \multicolumn{1}{c}{ POG } & \multicolumn{1}{c}{ PFOF } \\
\cline { 3 - 9 } & & 25 & 30 & 40 & 50 & 30 & 40 & 50 \\
\hline Trat. & 11 & $7,62 *$ & $9,64 *$ & $9,43 *$ & $5,56 *$ & $5,16 *$ & $9,43 *$ & $6,82 *$ \\
Bloque & 3 & $0,23 \mathrm{~ns}$ & $0,24 \mathrm{~ns}$ & $0,28 \mathrm{~ns}$ & $2,52 *$ & $0,28 \mathrm{~ns}$ & $0,28 \mathrm{~ns}$ & $1,48 \mathrm{~ns}$ \\
Error & 33 & 0,18 & 0,24 & 0,29 & 0,67 & 0,25 & 0,29 & 0,61 \\
Total & 47 & & & & & & & \\
CV $(\%)$ & & 7,74 & 8,52 & 9,15 & 11,89 & 9,31 & 9,15 & 11,99 \\
\hline
\end{tabular}

Trat.: Tratamientos; GL: Grados de libertad y CV: Coeficiente de variación.

* Significativo $(p \leq 0,05)$ y ns No Significativo $(p>0,05)$ de acuerdo con la prueba de F.

Tabla 2. Análisis de varianza para días a total germinación (DTG), índice de velocidad de germinación (IVG), índice de germinación (IG), días a total formación de fosforitos (DTFF), índice de la velocidad de formación de fosforitos (IVFF) e índice de formación de fosforitos (IFF) en el cultivo de café (Coffea arabica) var. Catuaí Rojo en Aragua de Maturín, estado Monagas, Venezuela.

\begin{tabular}{lccccccc}
\hline \multirow{2}{*}{$\begin{array}{c}\text { Fuente de } \\
\text { Variación }\end{array}$} & GL & \multicolumn{7}{c}{ Cuadrados medios } \\
\cline { 3 - 8 } & & DTG & IVG & IG & DTFF & IVFF & IFF \\
\hline Trat. & 11 & $163,24 *$ & $1,4129 *$ & $69143,0 *$ & $143,82 *$ & $1,049 *$ & $21165,7 *$ \\
Bloque & 3 & $6,51 \mathrm{~ns}$ & $0,1814 \mathrm{~ns}$ & $2137,1 \mathrm{~ns}$ & $4,50 \mathrm{~ns}$ & $0,091 \mathrm{~ns}$ & $1792,3 \mathrm{~ns}$ \\
Error & 33 & 14,96 & 0,0736 & 1719,9 & 16,161 & 0,04 & 1780,8 \\
Total & & & & & & & 123,22 \\
CV $(\%)$ & & 9,95 & 44,81 & 59,75 & 9,40 & 49,97 & 123,0 \\
\hline
\end{tabular}

Trat.: Tratamientos; GL: Grados de libertad y CV: Coeficiente de variación.

* Significativo $(\mathrm{p} \leq 0,05)$ y ns No Significativo $(\mathrm{p}>0,05)$ de acuerdo con la prueba de F. 
Tabla 3. Porcentaje de germinación a los 25, 30, 40 y 50 días después del soterrado de la semilla de café (Coffea arabica) var. Catuaí Rojo en Aragua de Maturín, estado Monagas, Venezuela.

\begin{tabular}{lcccc}
\hline \multirow{2}{*}{ Tratamientos } & \multicolumn{4}{c}{ Porcentaje de germinación } \\
\cline { 2 - 4 } & 25 & 30 & 40 & 50 \\
\hline ESC-MEC & $11,83 \mathrm{~B}$ & $21,95 \mathrm{~B}$ & $21,95 \mathrm{~B}$ & $47,81 \mathrm{~B}$ \\
ESC-MEC-IMN/24h & $69,67 \mathrm{~A}$ & $85,78 \mathrm{~A}$ & $88,84 \mathrm{~A}$ & $92,95 \mathrm{~A}$ \\
ESC-MEC-INM/48h & $77,45 \mathrm{~A}$ & $89,90 \mathrm{~A}$ & $90,94 \mathrm{~A}$ & $93,47 \mathrm{~A}$ \\
INM-AGUA/24h & $0,00 \mathrm{C}$ & $0,98 \mathrm{C}$ & $2,98 \mathrm{C}$ & $27,11 \mathrm{CD}$ \\
INM-AGUA/48h & $0,00 \mathrm{C}$ & $5,30 \mathrm{C}$ & $3,44 \mathrm{C}$ & $30,70 \mathrm{BCD}$ \\
INM-Ac.BAT/10s & $0,00 \mathrm{C}$ & $1,92 \mathrm{C}$ & $2,47 \mathrm{C}$ & $22,18 \mathrm{CD}$ \\
INM-Ac.BAT/20s & $0,00 \mathrm{C}$ & $1,46 \mathrm{C}$ & $4,17 \mathrm{C}$ & $21,59 \mathrm{CD}$ \\
INM-Ac.BAT/30s & $0,00 \mathrm{C}$ & $0,00 \mathrm{C}$ & $6,31 \mathrm{C}$ & $35,33 \mathrm{BC}$ \\
INM-Ac.MUR/10s & $0,00 \mathrm{C}$ & $1,48 \mathrm{C}$ & $1,48 \mathrm{C}$ & $12,17 \mathrm{D}$ \\
INM-Ac.MUR/20s & $0,00 \mathrm{C}$ & $0,00 \mathrm{C}$ & $1,48 \mathrm{C}$ & $20,33 \mathrm{CD}$ \\
INM-Ac.MUR/30s & $0,00 \mathrm{C}$ & $1,92 \mathrm{C}$ & $6,50 \mathrm{C}$ & $30,68 \mathrm{BCD}$ \\
Testigo & $0,00 \mathrm{C}$ & $1,48 \mathrm{C}$ & $1,98 \mathrm{C}$ & $18,33 \mathrm{CD}$ \\
MDS & 0,62 & 0,71 & 0,78 & 1,18 \\
\hline
\end{tabular}

ESC: Escarificación; MEC: Mecánica; INM: Inmersión; Ac.BAT: Ácido de batería $\left(\mathrm{H}_{2} \mathrm{SO}_{4}\right)$ y Ac.MUR: Ácido muriático (HCl). Porcentajes con letras diferentes dentro de una columna indican diferencias estadísticamente significativas de acuerdo con la prueba de la Mínima Diferencia Significativa $(\mathrm{p} \leq 0,05)$. MDS: se realizó a los datos transformados mediante $\mathrm{p}^{\prime}=\sqrt{(\mathrm{x}+3 / 8) /(\mathrm{n}+3 / 4)}$.

ambos tratamientos se explica que la ausencia del pergamino aumenta la germinación de la misma. Situación contraria presenta el testigo, el cual expresa un valor muy bajo, lo que reafirma de forma absoluta el retardo en la germinación de las semillas de café cuando son implementados los germinadores de manera tradicional por los productores, pues en estas condiciones la germinación oscila en un lapso de tiempo de 50 a 75 días. La exposición de las semillas con las técnicas de tratamiento con ácido muriático (INM-Ac.MUR/ 10, 20 y $30 \mathrm{~min}$.), ácido de batería (INM-Ac.BAT/ 10, 20 y 30 min.) e inmersión en agua durante 24 y 48 horas (INM-AGUA/24h y INM-AGUA/48h) no mostraron diferencias entre ellas, lo cual sugiere que la acción del ácido no fue lo suficientemente efectiva para la remoción del endocarpio. Asimismo, el tratamiento efectuado con la inmersión en agua no mejoró la germinación. El tratamiento representado por el testigo, el cual consistía en soterrar las semillas sin remoción del pergamino fue superior estadísticamente al tratamiento con ácido muriático con 10 minutos de inmersión.

\section{Formación de fosforitos}

Los tratamientos de escarificación mecánica con inmersión en agua durante 24 y 48 horas presentaron el mayor porcentaje de formación de fosforitos en todos los tiempos de evaluación (30,
40 y 50 días después de soterradas las semillas) (Tabla 4), superando al resto de los tratamientos. Los tratamientos de inmersión en agua, ácido de batería y ácido muriático presentaron porcentajes de formación de fosforitos similares al testigo en todos los tiempos de evaluación (Tabla 4). Estos resultados muestran la importancia que tiene la remoción del pergamino en las semillas de café para aumentar el porcentaje de formación de fosforitos, lo cual ratifica lo expresado por Guevara y Ramiro (1997) sobre la producción de plántulas con hipocotilos de mayor longitud cuando las semillas son escarificadas.

En las semillas, los fenómenos de germinación y verticalización del hipocotilo están ajustados a una sincronía y comportamiento lógico propio de la semilla (fenología), la cual puede ser afectada por factores externos. Los tratamientos que expresan un alto porcentaje de germinación son, a su vez, los tratamientos que manifiestan alto porcentaje de formación de fosforitos.

Urbaneja y Quijada (2006) en experimentos sobre la germinación de semillas de café encontraron que semillas tratadas con escarificación mecánica y otras tratadas por inmersión en agua durante 24 horas obtuvieron a los 36 días de observación un porcentaje de germinación de 90 y $93 \%$ respectivamente. En este caso se consideró como semilla germinada cuando la radícula emergía; sin embargo, en esta investigación con los tratamientos de escarificación mecánica con inmersión en agua durante 24 y 48 
Tabla 4. Porcentaje de formación de fosforitos a los 30, 40 y 50 días después del soterrado de la semilla de café (Coffea arabica) var. Catuaí Rojo en Aragua de Maturín, estado Monagas, Venezuela.

\begin{tabular}{lccc}
\hline & \multicolumn{2}{c}{ Tratamientos } & Porcentaje de formación de fosforitos \\
\cline { 2 - 4 } & 30 & 40 & 50 \\
\hline ESC-MEC & $6,69 \mathrm{~B}$ & $21,95 \mathrm{~B}$ & $33,21 \mathrm{~B}$ \\
ESC-MEC-IMN/24h & $53,06 \mathrm{~A}$ & $88,84 \mathrm{~A}$ & $91,42 \mathrm{~A}$ \\
ESC-MEC-INM/48h & $62,26 \mathrm{~A}$ & $90,94 \mathrm{~A}$ & $93,47 \mathrm{~A}$ \\
INM-AGUA/24h & $0,00 \mathrm{~B}$ & $2,98 \mathrm{C}$ & $17,80 \mathrm{BC}$ \\
INM-AGUA/48h & $0,00 \mathrm{~B}$ & $3,44 \mathrm{C}$ & $17,80 \mathrm{BC}$ \\
INM-Ac.BAT/10s & $0,00 \mathrm{~B}$ & $2,47 \mathrm{C}$ & $17,02 \mathrm{BC}$ \\
INM-Ac.BAT/20s & $0,00 \mathrm{~B}$ & $4,17 \mathrm{C}$ & $16,37 \mathrm{BC}$ \\
INM-Ac.BAT/30s & $0,00 \mathrm{~B}$ & $6,31 \mathrm{C}$ & $23,13 \mathrm{BC}$ \\
INM-Ac.MUR/10s & $0,00 \mathrm{~B}$ & $1,48 \mathrm{C}$ & $6,95 \mathrm{C}$ \\
INM-Ac.MUR/20s & $0,00 \mathrm{~B}$ & $1,48 \mathrm{C}$ & $12,39 \mathrm{BC}$ \\
INM-Ac.MUR/30s & $0,00 \mathrm{~B}$ & $6,50 \mathrm{C}$ & $22,45 \mathrm{BC}$ \\
TESTIGO & $0,00 \mathrm{~B}$ & $1,98 \mathrm{C}$ & $10,01 \mathrm{C}$ \\
MDS & 0,73 & 0,78 & 1,12 \\
\hline
\end{tabular}

ESC: Escarificación; MEC: Mecánica; INM: Inmersión; Ac.BAT: Ácido de batería $\left(\mathrm{H}_{2} \mathrm{SO}_{4}\right)$ y Ac.MUR: Ácido muriático (HCl). Porcentajes con letras diferentes dentro de una columna indican diferencias estadísticamente significativas de acuerdo con la prueba de la Mínima Diferencia Significativa $(\mathrm{p} \leq 0,05)$. MDS: se realizó a los datos transformados mediante $\mathrm{p}^{\prime}=\sqrt{(\mathrm{x}+3 / 8) /(n+3 / 4)}$.

horas se obtuvo a los 30 días (seis días menos) valores porcentuales de 85 y $89 \%$, respectivamente (Tabla 3). En este caso, se consideró de manera absoluta semillas germinadas en la que emergía el hipocotilo en forma de gancho invertido. Lo que permite distinguir que el tiempo empleado para la emergencia de la radícula es sustancialmente reducido toda vez que en menor tiempo se revela la presencia del hipocotilo; además, la formación de fosforitos se presenta en un tiempo radicalmente corto en ambos tratamientos, ya que a los 40 días de observación se obtuvieron porcentajes de 88 y $90 \%$ en los tratamientos antes mencionados (Tabla 4).

Finalmente se considera que la germinación en las semillas de café tiene un lapso comprendido entre 34 y 45 días, y la formación de fosforitos tarda un tiempo de 60 a 75 días, en este trabajo se observó una reducción significativa de 25 días para la germinación y 30 días para la formación del fosforito en el proceso de las labores de germinador.

\section{Número días de germinación, índice de la velocidad de germinación e índice de germinación}

Esta variable y las dos subsiguientes (IVG e IG) consolidan la efectividad de los tratamientos en donde se emplea el uso de la escarificación mecánica más el remojo en agua durante 24 y 48 horas al permitir obtener los resultados más eficientes en acelerar la germinación de las semillas (Tabla 5). Resultados similares indicó Camacho Llaguno (1991) al estudiar el efecto de los bioestimulantes sobre la germinación de las semillas y desarrollo vegetativo de las plántulas de café y concluyeron que los bioestimulantes Triggrr, Ergostim y Agrispon, no tuvieron efecto sobre la germinación de la semilla ni el desarrollo vegetativo de las plántulas pero las semillas desprovistas del pergamino germinaron más rápidamente, pero estuvieron más propensas al damping off.

Los tiempos empleados para la germinación para cada tratamiento (Tabla 5) agrupados por su resultado estadístico; en donde los tratamientos ESC-MEC-48H y ESC-MEC-48H alcanzaron el menor número de días para experimentar el fenómeno de germinación, los otros tratamientos fueron estadísticamente diferentes a estos. Es decir, existe una estrecha relación entre los valores porcentuales de germinación de la semilla y el tiempo empleado para ello, pues los tratamientos antes mencionados experimentaron altos valores de germinación y el menor tiempo para el efecto de este fenómeno.

La comparación de las medias de los días para que sucediera la germinación agrupó a los tratamientos inmersión con ácido muriático en un tiempo de 10 y 30 minutos, inmersión en agua durante 24 y 48 horas e inmersión en ácido de batería 30 minutos como 
Tabla 5. Días a total germinación (DTG), índice de la velocidad de germinación (IVG) e índice de germinación (IG) de la semilla de café (Coffea arabica) var. Catuaí Rojo en Aragua de Maturín, estado Monagas, Venezuela

\begin{tabular}{lllr}
\hline \multicolumn{1}{c}{ Tratamientos } & DTG & IVG & IG \\
\hline ESC-MEC & $36,70 \mathrm{~B}$ & $0,76 \mathrm{~B}$ & $84,67 \mathrm{~B}$ \\
ESC-MEC-IMN/24h & $26,70 \mathrm{~A}$ & $1,77 \mathrm{~A}$ & $328,15 \mathrm{~A}$ \\
ESC-MEC-INM/48h & $25,20 \mathrm{~A}$ & $1,90 \mathrm{~A}$ & $364,23 \mathrm{~A}$ \\
INM-AGUA/24h & $39,80 \mathrm{BC}$ & $0,35 \mathrm{C}$ & $5,28 \mathrm{C}$ \\
INM-AGUA/48h & $40,50 \mathrm{BC}$ & $0,40 \mathrm{BC}$ & $14,72 \mathrm{C}$ \\
INM-Ac.BAT/10s & $42,90 \mathrm{C}$ & $0,29 \mathrm{C}$ & $6,42 \mathrm{C}$ \\
INM-Ac.BAT/20s & $42,60 \mathrm{C}$ & $0,28 \mathrm{C}$ & $5,60 \mathrm{C}$ \\
INM-Ac.BAT/30s & $41,80 \mathrm{BC}$ & $0,45 \mathrm{BC}$ & $4,28 \mathrm{C}$ \\
INM-Ac.MUR/10s & $41,80 \mathrm{BC}$ & $0,15 \mathrm{C}$ & $4,20 \mathrm{C}$ \\
INM-Ac.MUR/20s & $44,80 \mathrm{C}$ & $0,26 \mathrm{C}$ & $2,25 \mathrm{C}$ \\
INM-Ac.MUR/30s & $41,00 \mathrm{BC}$ & $0,43 \mathrm{BC}$ & $8,40 \mathrm{C}$ \\
TESTIGO & $42,90 \mathrm{C}$ & $0,22 \mathrm{C}$ & $4,77 \mathrm{C}$ \\
MDS & 5,57 & 0,39 & 59,66 \\
\hline
\end{tabular}

ESC: Escarificación; MEC: Mecánica; INM: Inmersión; Ac.BAT: Ácido de batería $\left(\mathrm{H}_{2} \mathrm{SO}_{4}\right)$ y Ac.MUR: Ácido muriático (HCl). Porcentajes con letras diferentes dentro de una columna indican diferencias estadísticamente significativas de acuerdo con la prueba de la Mínima Diferencia Significativa $(\mathrm{p} \leq 0,05)$.

estadísticamente iguales entre sí pero diferentes a los tratamientos de escarificación. Lo que sugiere que la utilización de ácidos y el empleo de sumergir las semillas en agua tienen efectos similares, pues no llegan a eliminar de forma eficiente la epidermis. En consecuencia, se deberán incrementar los tiempos de inmersión de las semillas cuando se empleen estos tratamientos para ver si pueden eliminar el endosperma. El tratamiento testigo empleó un tiempo mayor de 42 días para la germinación corroborando el retardo de la germinación de las semillas de café que se observa en los germinadores construidos por los productores, los cuales tienen un periodo de tiempo de 45 a 60 días.

El porcentaje y el tiempo de germinación fueron afectados positivamente por el índice de velocidad de germinación en los tratamientos ESC-MEC-INM por 24 y $48 \mathrm{~h}$, toda vez que el mayor índice de velocidad (1,90 y 1,77 respectivamente) se registra en estos dos tratamientos como lo muestra la Tabla 5. Por otro lado, se tiene que el índice de velocidad del testigo es uno de los más bajos con 0,22 , lo cual guarda coherencia con su bajo porcentaje y días de germinación manifestado.

Número de días para la formación de "fosforitos", índice de la velocidad de formación de fosforitos e índice de la formación de fosforitos

Hay diferencias significativas entre los tratamientos ESC-MEC-INM por 24 y $48 \mathrm{~h}$, con el resto de los tratamientos en relación con los días empleados para la formación de fosforitos, así como los dos índices evaluados (Tabla 6). Estos tienen un mayor porcentaje de formación de fosforitos en un tiempo menor de 31, 30 días, lo cual evidencia el efecto de la escarificación mecánica o destrucción del pericarpio de las semillas y su inmersión inmediata en agua durante 24 y 48 horas, en condiciones normales, el tiempo empleado para la formación de fosforitos es mayor de 50 días. Por otro lado, los demás tratamientos fueron homogéneos pues expresaron valores en un rango que va de 43,80 días y menores de 46,10 días, salvo el tratamiento inmersión en ácido muriático durante 20 minutos que experimentó un valor de 52,10 días. Además, para esta evaluación, el testigo se comportó de manera similar al resto de los tratamientos empleado para la formación de los fosforitos un tiempo medio mayor de 44 días.

El índice medio de la velocidad para la formación de fosforitos fue mayor en los tratamientos de escarificación mecánica e inmersión en agua en 24 y 48 horas con una media de 1,48 y 1,54 , respectivamente, siendo estos valores estadísticamente semejantes, pero al compararse a los valores expresados por el resto de los tratamientos son diferentes. Resultados similares se encontraron para el índice de la velocidad de formación de fosforitos (Tabla 6). Lo cual establece que bajo estos métodos de escarificación combinada hay una aceleración significativa en la formación de esta estructura vegetal en un tiempo menor. Lo 
Tabla 6. Días a total formación de fosforitos (DTFF), índice de la velocidad de formación de fosforitos (IVFF) e índice de la formación de fosforitos (IFF) de la semilla de café (Coffea arabica) var. Catuaí Rojo en Aragua de Maturín, estado Monagas, Venezuela.

\begin{tabular}{lllr}
\hline \multicolumn{1}{c}{ Tratamientos } & DTFF & IVFF & IFF \\
\hline ESC-MEC & $45,20 \mathrm{~B}$ & $0,48 \mathrm{~B}$ & $23,13 \mathrm{~B}$ \\
ESC-MEC-IMN/24h & $31,30 \mathrm{~A}$ & $1,49 \mathrm{~A}$ & $153,85 \mathrm{~A}$ \\
ESC-MEC-INM/48h & $31,10 \mathrm{~A}$ & $1,54 \mathrm{~A}$ & $211,85 \mathrm{~A}$ \\
INM-AGUA/24h & $42,80 \mathrm{~B}$ & $0,22 \mathrm{BC}$ & $1,50 \mathrm{~B}$ \\
INM-AGUA/48h & $44,50 \mathrm{~B}$ & $0,21 \mathrm{BC}$ & $1,38 \mathrm{~B}$ \\
INM-Ac.BAT/10s & $45,90 \mathrm{~B}$ & $0,20 \mathrm{BC}$ & $1,23 \mathrm{~B}$ \\
INM-Ac.BAT/20s & $46,10 \mathrm{~B}$ & $0,20 \mathrm{BC}$ & $1,35 \mathrm{~B}$ \\
INM-Ac.BAT/30s & $43,80 \mathrm{~B}$ & $0,27 \mathrm{BC}$ & $2,00 \mathrm{~B}$ \\
INM-Ac.MUR/10s & $45,20 \mathrm{~B}$ & $0,08 \mathrm{C}$ & $0,47 \mathrm{~B}$ \\
INM-Ac.MUR/20s & $52,10 \mathrm{C}$ & $0,16 \mathrm{C}$ & $1,10 \mathrm{~B}$ \\
INM-Ac.MUR/30s & $44,30 \mathrm{~B}$ & $0,29 \mathrm{BC}$ & $2,23 \mathrm{~B}$ \\
TESTIGO & $44,90 \mathrm{~B}$ & $0,13 \mathrm{C}$ & $0,90 \mathrm{~B}$ \\
MDS & 5,78 & 0,31 & 60,71 \\
\hline
\end{tabular}

ESC: Escarificación; MEC: Mecánica; INM: Inmersión; Ac.BAT: Ácido de batería $\left(\mathrm{H}_{2} \mathrm{SO}_{4}\right)$ y Ac.MUR: Ácido muriático (HCl). Porcentajes con letras diferentes dentro de una columna indican diferencias estadísticamente significativas de acuerdo con la prueba de la Mínima Diferencia Significativa $(\mathrm{p} \leq 0,05)$.

cual sugiere por un lado el empleo de esta técnica combinada y por otro una revisión de los otros tratamientos. El testigo expresa un índice medio de velocidad de 0,125 , el cual es extremadamente bajo cuando se le compara estadísticamente con los dos tratamientos que arrojaron mayor índice de velocidad (Tabla 6).

Finalmente el método de escarificación mecánica combinados con el método de remojo en agua durante 24 y 48 horas fue superior al resto de los tratamientos tanto en la etapa de germinación como en la etapa de formación de fosforito; asimismo, el análisis de las variables empleadas para su estudio expresan los mejores valores de rendimiento para estos dos tratamientos. Valio (1980) indicó que la presencia del endocarpo inhibe drásticamente la germinación de las semillas de café. Los resultados obtenidos en este trabajo están en concordancia a los obtenidos por Valio (1980), lo que ratifica las bondades del proceso de escarificación mecánica y la inmersión en agua de las semillas de café por 24 o 48 previas al soterrado. Al respecto, Fontanibe (1987) reportó que el pergamino no influye en la imbibición de agua, pero posiblemente constituye una barrera a la difusión del oxígeno, provocando un retardo y una disminución de la germinación total y que los más altos porcentajes de germinación estuvieron asociados a los tratamientos de escarificación total del pergamino con el escarificador eléctrico y a la atmósfera de $60 \%$ de oxígeno, mientras que la escarificación mecánica con el despulpador Bentall removió efectivamente el pergamino de la semilla, pero causa daño al embrión y la inmersión de las semillas en agua hirviendo afectó al embrión causando la pérdida total de la germinación. El testigo se manifestó en las fases de germinación y formación de fosforito con valores similares a los observados normalmente cuando se realizan de manera tradicional los germinadores en las zonas cafetaleras del estado Monagas, mientras que Figueroa Núñez (1982) estudió la escarificación de la semilla de café Caturra, mediante tratamientos físicos y químicos para acelerar la germinación y reportó que el mejor promedio de germinación lo obtuvo el tratamiento supresión total del pergamino, estadísticamente igual al tratamiento testigo y agua hirviendo, en cuanto a la altura de planta, no se observó una diferencia significativa sobre los tratamientos con relación al testigo; además que la supresión total del pergamino tuvo la mayor altura de planta a los 80 días.

En este experimento, la imbibición de las semillas de café en agua durante 24 y 48 horas no promovió la germinación y la formación de fosforitos y tuvo resultados similares al testigo. Al respecto, Alava Moreira y Vergara Reyes (1990) realizaron un experimento para estudiar los efectos de inhibir semillas de café con pergamino en agua destilada, empleándose cuatro variedades de café: Caturra, Catimor Populaçao 3, Vermelho UFV-2237 y Pacas y tiempos de imbibición de $0,12,24$ y 36 horas y 
encontraron que la variedad Catimor Populaçao 3 presentó el mayor porcentaje de germinación, pero ninguna variedad alcanzó el 100\% de germinación e indicaron que la constitución física del pergamino y las variaciones de temperatura del agua en que se inhibieron las semillas de café influyeron en su porcentaje de germinación, recomendando someter a las semillas de café a imbibición en agua natural, para alcanzar un mayor porcentaje de imbibición. Adicionalmente no se observó una respuesta positiva a la inmersión en ácido de batería $\left(\mathrm{H}_{2} \mathrm{SO}_{4}\right)$ ni ácido muriático $(\mathrm{HCl})$ por 10, 20 y 30 segundos. Similares resultados fueron señalados por Figueroa Núñez (1982) quien indicó que hubo una completa nulidad en los tratamientos con ácido nítrico al 30 y $60 \%$, por inhibir totalmente la germinación según se observó desde los 35 hasta los 55 días.

Como se ha mencionado anteriormente, los mejores tratamientos fueron escarificación mecánica e inmersión en agua durante 24 y 48 horas, mientras los tratamientos de inmersión en agua, ácido de batería y ácido muriático no fueron efectivos en promover la germinación y formación de fosforitos, ni tampoco para acelerar la germinación. Resultados diferentes indicaron Molina Arcentales et al. (1981) quienes emplearon semillas de café de variedad Caturra, las mismas que se pusieron en imbibición por el lapso de 30 minutos, en los ácidos nítrico, clorhídrico y sulfúrico y 30 y 60 segundos en el agua hirviendo, mientras que los tratamientos supresión del pergamino y testigo no recibieron ningún tratamiento químico e indicaron que en la primera fase, los tratamientos que produjeron la mortalidad del embrión de las semillas fueron ácido nítrico al 50 y $75 \%$, ácido clorhídrico al $75 \%$ y agua hirviendo, siendo estos tratamientos descartados en la segunda prueba, para la segunda fase, el ácido clorhídrico al $25 \%$ fue el tratamiento que produjo los más altos valores de germinación hasta los 40 días, aunque también dieron buenos resultados en este periodo los tratamientos de ácido clorhídrico al $50 \%$, ácido sulfúrico al $25 \%$ y supresión del pergamino.

\section{Conclusiones}

El uso de las técnicas de escarificación mecánica a las semillas de café acelera su germinación y la formación de fosforitos.

Los tratamientos donde las semillas de café se sometieron a escarificación mecánica más inmersión en agua durante 24 y 48 horas presentaron los mayores valores de germinación y formación de fosforitos. Sesenta por ciento de la germinación ocurrió a los 25 días y la formación de fosforitos ocurrió a los 30 días de soterradas las semillas.

Los tratamientos de escarificación química empleando los productos comerciales con ácido muriático (Opinß) y de batería (Copper®) no ofrecieron ventajas con relación al testigo.

\section{Literatura Citada}

Alava Moreira, G.M.; Vergara Reyes, M.M.

1990. Efectos de 4 tiempos de imbibición en agua destilada de la semilla de 4 variedades de café en su germinación. Tesis para Ingeniero Agrícola. Facultad de Ingeniería Agrícola, Universidad Técnica de Manabi. 47 pp.

Amaya L.F.; Celis, B.; Farrera P.R.; García, A.M.; Manrique M.J.; Medina, J.; Murillo, A.; Romero, A.; Sánchez, L.; Sayago

A.M.; Silva-Acuña, R.; Yánez, P.; Zavala, Y.

1988. Paquete tecnológico para la producción de café. FONAIAP. Maracay, Venezuela. 192 pp.

Camacho Llaguno, Z.L.

1991. Efectos de los bioestimulantes en semilleros y viveros del café Coffea arabica L. variedad Caturra en la zona de Ventanas. Tesis para Ingeniero Agrónomo. Facultad de Ciencias Agrícolas, Universidad Técnica de Babahoyo, 36 pp.

Da Silva, E.A.A.

2002. Coffee (Coffea arabica cv. Rubi) seed germination: mechanism and regulation. PhD Thesis. Wageningen, Wageningen Agricultural University.
Eira, M.T.S.; Amaral da Silva, E.A.; De Castro, R.D.; Dussert, S.; Walters, C.; Derek Bewley, J.; Hilhorst, H.W.M.

2006. Coffee seed physiology. Braz. J. Plant Physiol 18 (1): 149-163.

Figueroa Núñez, S.L.

1982. Escarificación de la semilla de café Caturra mediante tratamientos físicos y químicos para acelerar la germinación. Trabajo de Grado para Ingeniero Agrónomo. Facultad de Agronomía, Universidad de Guayaquil. 32 pp.

Fontanibe V., P.A.

1987. Efecto de tratamientos pregerminativos en semillas de café (Coffea arabica L.). Trabajo de grado para Magister Scientiarum en Agronomía, Universidad Central de Venezuela.

Guevara E.; Herrera, J.; Ramiro, A.

1992. Efecto de la cianamida hidrogenada en la semilla de café (Coffea arabica) cv. Caturra. II. Influencia sobre el metabolismo germinativo. Agronomía Costarricense 16 (2): 177-185. 
Guevara E.; Herrera, J.; Ramiro, A.

1997. Efecto del sustrato y su condición hídrica sobre la germinación de semillas de café Caturra. Agronomía Costarricense, 21 (2): 207-216.

Izco, J.; Barreno, E.; Brugues, M.; Costa, M.; Devesa, J.; Fernández, F.; Talavera, S.; Valdéz, B. 1997. Botánica. McGraw-HillInteramericana de España. Madrid, España. 781 p. 1997. Botánica, Primera Edición. España.

Madueño, A.; García, D.; Hernández, J.; Rubio, C.; Navarrete, A.; Bojorquez, J.

2006. Germinación de semillas de frijolillo, Rhynchosia minima (L) DC., luego de someterla a tratamientos de pregerminación Bioagro, 18 (2): 101-105.

Maguire, J.D.

1962. Speed of germination-aid in selection and evaluation for seedling emergence and vigor. Crop Science, 2 (2): 176-177.

Manuales para Educación Agropecuaria (MEA). 1983. Cultivo de Plantación. Área Producción Vegetal. Trillas. México. 25-40.

Medina, J.; Sayago, M.; Farrera, R.; García, M.; Amaya, F., Silva-Acuña, R.; Morócoima, J.; Zabala, Y. 1987. Manual de alternativa tecnológica para el cultivo de café a Nivel de campesino en Venezuela. FONAIAP. MaracayVenezuela. $157 \mathrm{pp}$.
Moreno Martínez, M.E.

1996. Análisis físico y biológico de semillas agrícolas. Instituto de Biología. Universidad Nacional Autónoma de México (UNAM). México 393 p.

Molina Arcentales, V.S.; Bowen C.T.; Solorzano Loor, M.M. 1981. Escarificación de la semilla del café Caturra mediante tratamientos físicos y químicos para acelerar la germinación. Trabajo de Grado Ingeniero Agrónomo. Facultad de Ingeniería Agronómica, Universidad Técnica de Manabi. 38 pp.

Rincón. S.O.

1982. Manual para el cultivo del café. Manual Práctico. $3^{\mathrm{a}}$ edición. Bogotá-Colombia.

Sanabria, D.; Silva-Acuña, R.; Oliveros, M.; Manrique, U. 2004. Germinación de semillas de las leguminosas arbustivas forrajeras Cratylia argentea y Cassia moschata sometidas a inmersión en ácido sulfúrico. Bioagro, 16 (3): 225-230. Solomon, E.; Berg, K.; D. Martín, D.

2001. Biología. $5^{\text {ta }}$ Edición. McGraw-Hill Interamericana, México. 1237 p.

Urbaneja S.; Quijada, M.

2006. Evaluación de diferentes tratamientos en pregerminación de café para la obtención de plántulas. Proyecto de Investigación Rubro Café. Instituto Nacional de Investigaciones Agrícolas, Caripe, estado Monagas, Venezuela. 38 p.

Valio, I.F.M.

1980. Inhibition of germination of coffee seeds (Coffea arabica L. cv. Mundo Novo) by the endocarp. J. Seed Technol. 5: 32-39. 
